\title{
THE FRANK NELSON COLE PRIZE IN ALGEBRA
}

The first award of the Cole prize will be made at the end of 1927 for a notable contribution to the theory of linear algebras by a resident of the United States or Canada.

1. The contribution may be either an unpublished manuscript or a paper first published during 1925, 1926, or 1927.

2. Two copies of the manuscript or printed paper should be in the hands of the Secretary of the Society, 501 West 116 th Street, New York City, on or before October 31, 1927.

3 . The first award will be two hundred dollars $(\$ 200,00)$.

4. The prize will be withheld in the absence of a sufficiently meritorious memoir.

Without excluding others, the following topics are suggested as suitable ones:

a. Determination of all division algebras of rank 5 . (For those of rank 4, see F. Cecioni, Palermo Rendiconti, vol. 47 [1923], pages 209-25̃4.)

b. Direct proof, without the theory of the rank equation, that an algebra whose units form a group is semi-simple.

c. Classification of nilpotent algebras.

d. Every algebra $A$ is the sum of a semi-simple subalgebra $S$ and the maximal nilpotent invariant sub-algebra $N$. Investigate the multiplicative relations between units of $S$ and units of $N$.

e. Develop a rational theory of algebras $A$ over any field by starting with the rank function of the general element of $A$ expressed in terms of the rank functions of the simple components of $S$ in problem $d$.

f. Extend the theory of arithmetics of algebras. In particular, invent a theory of ideals for division algebras.

The literature is accessible in G. Scorza's Corpi Numerici e Algebre, Messina, 1921, and Dickson's Algebras and their Arithmetics, University of Chicago Press, 1923.

\section{H. S. White,}

For the Committee of the Council. 